\title{
ON THE SOLUTION OF THE CONTINUITY EQUATION FOR PRECIPITATING ELECTRONS IN SOLAR FLARES
}

\author{
A. Gordon EMSLIE ${ }^{1}$, Gordon D. Holman ${ }^{2}$, ANd Yuri E. LitvinenKo ${ }^{3}$ \\ ${ }^{1}$ Department of Physics \& Astronomy, Western Kentucky University, Bowling Green, KY 42101, USA; emslieg@wku.edu \\ ${ }^{2}$ Code 671, NASA Goddard Space Flight Center, Greenbelt, MD 20771, USA; gordon.d.holman@ nasa.gov \\ ${ }^{3}$ Department of Mathematics, University of Waikato, P. B. 3105, Hamilton, New Zealand \\ Received 2014 June 19; accepted 2014 July 17; published 2014 August 7
}

\begin{abstract}
Electrons accelerated in solar flares are injected into the surrounding plasma, where they are subjected to the influence of collisional (Coulomb) energy losses. Their evolution is modeled by a partial differential equation describing continuity of electron number. In a recent paper, Dobranskis \& Zharkova claim to have found an "updated exact analytical solution" to this continuity equation. Their solution contains an additional term that drives an exponential decrease in electron density with depth, leading them to assert that the well-known solution derived by Brown, Syrovatskii \& Shmeleva, and many others is invalid. We show that the solution of Dobranskis \& Zharkova results from a fundamental error in the application of the method of characteristics and is hence incorrect. Further, their comparison of the "new" analytical solution with numerical solutions of the Fokker-Planck equation fails to lend support to their result. We conclude that Dobranskis \& Zharkova's solution of the universally accepted and well-established continuity equation is incorrect, and that their criticism of the correct solution is unfounded. We also demonstrate the formal equivalence of the approaches of Syrovatskii \& Shmeleva and Brown, with particular reference to the evolution of the electron flux and number density (both differential in energy) in a collisional thick target. We strongly urge use of these long-established, correct solutions in future works.
\end{abstract}

Key words: methods: analytical - plasmas - Sun: atmosphere - Sun: flares - Sun: particle emission Sun: X-rays, gamma rays

\section{INTRODUCTION}

The hard X-ray emission from solar flares is generally well described by the thick-target model (Brown 1971), in which deka-keV electrons are accelerated in the solar corona and stream downward to the denser regions of the solar atmosphere where they collisionally lose their energy, radiating intense bremsstrahlung X-rays and heating the flare plasma in the process (e.g., Holman et al. 2011; Kontar et al. 2011). The evolution of the electron spectrum with distance from the acceleration region is, for most purposes, adequately described by the analytical solution of the one-dimensional (1D) continuity equation with Coulomb collisional energy losses (Syrovatskii \& Shmeleva 1972).

In a recent paper, Dobranskis \& Zharkova (2014, hereafter DZ) claim (their Section 2) that the existing analytical solution (Brown 1971; Syrovatskii \& Shmeleva 1972) is "not mathematically correct," and specifically (in their abstract) that the continuity equation has been solved "ignoring an additional free term with an electron density." Using the method of characteristics to solve the continuity equation for the electron density distribution function $N(E, s)$ (electrons $\left.\mathrm{cm}^{-3} \mathrm{keV}^{-1}\right)$, they claim that the solution contains a hitherto unrecognized exponential term that substantially decreases the density of electrons, especially at low energies. They also compare their solution with numerical Fokker-Planck (FP) results and argue that this comparison provides a verification of their analytical solution.

If true, the claims of DZ would have major implications, inasmuch as the original thick target model solution (Brown 1971; Syrovatskii \& Shmeleva 1972) and its more sophisticated versions (e.g., Brown 1972; Leach \& Petrosian 1981; McClements 1992) are routinely used to deduce energetic electron distributions from flare hard X-ray data (e.g., Holman et al. 2003; Kontar et al. 2011 and references therein). However, we demonstrate here that there is an essential mathematical error in the DZ analysis that renders their basic result, and hence all their subsequent conclusions, invalid. When this error is corrected (Section 2.1), the well-established existing solution is recovered. For completeness, in Section 2.2 we show the complete equivalence of the continuity equation formalisms of Brown (1971) and Syrovatskii \& Shmeleva (1972), and that they both lead to the same well-established solution. In Section 2.3, we further argue that comparison with numerical results provides ample support for the well-established existing solution (contrary to claims by DZ). We conclude in Section 3 that the solution of Dobranskis \& Zharkova (2014) is fundamentally flawed and that the conventional wisdom stands.

\section{CRITIQUE OF THE DOBRANSKIS \& ZHARKOVA RESULTS}

\subsection{Analytical Solution}

DZ consider the $1 \mathrm{D}$ continuity equation

$$
\frac{\partial F(E, s)}{\partial s}+\frac{\partial}{\partial E}\left[\left(\frac{d E}{d s}\right) F(E, s)\right]=0
$$

for the electron flux $F(E, s)$ (electrons $\mathrm{cm}^{-2} \mathrm{~s}^{-1} \mathrm{keV}^{-1}$ ), differential in energy $E(\mathrm{keV})$, at position $s(\mathrm{~cm})$ from the location at which the electrons are injected (the acceleration region). They then set $F(E, s)=N(E, s) v(E)$, where $N(E, s)$ is the electron density distribution function (electrons $\mathrm{cm}^{-3} \mathrm{keV}^{-1}$ ) and $v=\sqrt{2 E / m_{e}}$ is the electron speed $\left(\mathrm{cm} \mathrm{s}^{-1}\right)$. This gives their Equation (11), the continuity equation for $N(E, s)$ :

$$
\frac{\partial}{\partial s}\left[\sqrt{\frac{2 E}{m_{e}}} N(E, s)\right]+\frac{\partial}{\partial E}\left[\left(\frac{d E}{d s}\right) \sqrt{\frac{2 E}{m_{e}}} N(E, s)\right]=0 .
$$


For an energy loss term appropriate to Coulomb collisions (Equation (2) of DZ),

$$
\frac{d E}{d s}=-\frac{a n}{E}
$$

where $n$ is the ambient density $\left(\mathrm{cm}^{-3}\right)$ and $a$ is a constant (for details see Syrovatskii \& Shmeleva 1972), the continuity Equation (2) reduces to the form (Equation (13) of DZ)

$$
\frac{\partial N}{\partial \xi}-\frac{a}{E} \frac{\partial N}{\partial E}=-\frac{a N}{2 E^{2}},
$$

where the column density $\xi(s)=\int_{0}^{s} n\left(s^{\prime}\right) d s^{\prime}$.

The method of characteristics is a very useful tool in solving partial differential equations such as (4). Indeed, Craig et al. (1985) have used this method to solve a more general kinetic equation that describes the evolution of a time-dependent anisotropic distribution of energetic electrons. Using the method of characteristics, DZ correctly reduce Equation (4) to a set of three first-order, coupled ordinary differential equations in terms of a parameter $t$ (Equations (15) through (17) of DZ):

$$
\begin{aligned}
& \frac{d \xi}{d t}=1 \\
& \frac{d E}{d t}=-\frac{a}{E} ; \\
& \frac{d N}{d t}=-\frac{a N}{2 E^{2}} .
\end{aligned}
$$

Equation (5) simply states that the parameter $t$ is equal to the column density $\xi$, in agreement with DZ. Given this result, the second equation (6) gives the well-known energy evolution of a single electron as a function of column density:

$$
E=\sqrt{E_{0}^{2}-2 a \xi}
$$

where $E_{0}$ (the injected energy) is a constant, also in agreement with DZ. However, in solving the final equation (7), DZ make a significant error. Treating $E$ as a constant, they erroneously infer that $\ln N+a t / 2 E^{2}=$ const is an integral of Equation (7) and hence that

$$
N=N_{0}\left(E_{0}\right) e^{-a t / 2 E^{2}} .
$$

With the identifications $\xi=t$ (Equation (5)) and $E_{0}=$ $\sqrt{E^{2}+2 a \xi}$ (Equation (8)), this leads to the overall "solution" (their Equation (18))

$$
N(E, \xi)=\Psi\left(\xi+\frac{E^{2}}{2 a}\right) e^{-a \xi / 2 E^{2}}
$$

where $\Psi$ is related to the form of the injected spectrum $N_{0}$.

It must first be observed that the "solution" (10) does not satisfy the continuity equation (4). To see this, we note that the partial derivatives of Equation (10) with respect to $\xi$ and $E$ are, respectively,

$$
\frac{\partial N}{\partial \xi}=\Psi^{\prime} e^{-a \xi / 2 E^{2}}-\frac{a}{2 E^{2}} N
$$

and

$$
\frac{\partial N}{\partial E}=\frac{E}{a} \Psi^{\prime} e^{-a \xi / 2 E^{2}}+\frac{a \xi}{E^{3}} N
$$

Substituting these into Equation (4) gives

$$
\Psi^{\prime} e^{-a \xi / 2 E^{2}}-\frac{a}{2 E^{2}} N-\Psi^{\prime} e^{-a \xi / 2 E^{2}}-\frac{a^{2} \xi}{E^{4}} N \stackrel{?}{=}-\frac{a}{2 E^{2}} N,
$$

leaving an unbalanced term $\left(a^{2} \xi / E^{4}\right) N$ on the left side. From a physical perspective, failure of the "solution" (10) to satisfy the continuity equation (4) implies non-conservation of electron number, and hence momentum, electrical current, and energy, all of which represent fatal flaws.

To obtain the correct solution of the characteristic equation (7), we note that, contrary to the assumption made by DZ, $E$ is not independent of $\xi=t$, as Equation (8) clearly shows. With this explicit dependence taken into account, Equation (7) may be written

$$
\frac{d N}{N}=-\frac{a d t}{2\left(E_{0}^{2}-2 a t\right)} .
$$

This integrates to

$$
N(E, \xi)=\frac{E^{1 / 2}}{\left(E^{2}+2 a \xi\right)^{1 / 4}} N_{0}\left(\sqrt{E^{2}+2 a \xi}\right)=E^{1 / 2} \Phi\left(\xi+\frac{E^{2}}{2 a}\right),
$$

where $N_{0}\left(E_{0}\right)$ is the electron density distribution function injected at $\xi=0$ and $\Phi$ is defined accordingly. This is consistent with the solution found by previous authors (see Equations (4) and (6) in Syrovatskii \& Shmeleva 1972). It is also readily verified that Equation (15) is the (unique) solution of the continuity equation (4).

\subsection{Comparison with Previous Treatments}

The differential form (1) used by DZ, Syrovatskii \& Shmeleva (1972), Emslie et al. (2001), and others for the continuity equation (particle conservation) at first sight looks very different from the integral form

$$
F(E) d E=F\left(E_{0}\right) d E_{0}
$$

used by, e.g., Brown (1971, 1972), Emslie (1978) and many others. To eliminate any risk that these different formalisms might be thought of as giving rise to different physical descriptions of electron beam propagation, we show here that they are precisely equivalent.

For a general form of the energy loss rate

$$
\frac{d E}{d s}=g(E, s)
$$

the differential continuity equation (1) becomes

$$
\frac{\partial F(E, s)}{\partial s}+g(E, s) \frac{\partial F(E, s)}{\partial E}=-\frac{\partial g(E, s)}{\partial E} F(E, s) .
$$

The characteristic equations for this partial differential Equation are

$$
\frac{d s}{d t}=1 ; \quad \frac{d E}{d t}=g(E, s) ; \quad \frac{d F}{d t}=-\frac{\partial g(E, s)}{\partial E} F .
$$

The first of these simply identifies $t=s$. Combining the second and third gives

$$
\frac{d F}{F}=-\frac{\partial g(E, s) / \partial E}{g(E, s)} d E .
$$


This integrates to

$$
\frac{F(E)}{F_{0}\left(E_{0}\right)}=\frac{g\left(E_{0}, s\right)}{g(E, s)}=\frac{d E_{0}}{d E},
$$

where the last equality uses Equation (17). The result (21) precisely corresponds to the integral formalism of Equation (16), and simply expresses the equality of the electron flux in the energy range $[E, E+d E]$ and the injected flux in the corresponding energy range $\left[E_{0}, E_{0}+d E_{0}\right]$. It should be noted that $d E_{0} / d E$ is evaluated locally, i.e., it is the ratio of the energy loss rate an electron of energy $E_{0}$ would experience at position $s$ to the energy loss rate experienced by an electron of energy $E$ at the same position $s$.

For the special case of Coulomb collisional losses, $d E / d \xi=$ $-a / E$ (Equation (3) and the definition of column density, $\xi$ ), the flux continuity equation (1) may be reduced to the form

$$
\frac{\partial F(E, \xi)}{\partial \xi}-\frac{a}{E} \frac{\partial F(E, \xi)}{\partial E}=-\frac{a F(E, \xi)}{E^{2}},
$$

a form similar to Equation (4) (and Equation (13) of DZ), but lacking the factor 2 in the denominator of the term on the right side because of the essential difference in the quantities $N$ and $F$. As in Section 2.1, Equation (22) may be solved by the method of characteristics to yield the solution

$$
F(E, \xi)=\frac{E}{\left(E^{2}+2 a \xi\right)^{1 / 2}} F_{0}\left(\sqrt{E^{2}+2 a \xi}\right) .
$$

We next consider the integral formalism (16). From Equation (8), taking differentials at a fixed value of $\xi$ (see discussion after Equation (21)), $2 E d E=2 E_{0} d E_{0}$, so that $d E_{0} / d E=E / E_{0}$. Using this in Equation (21), we obtain

$$
F(E, \xi)=\frac{E}{E_{0}} F_{0}\left(E_{0}\right)=\frac{E}{\left(E^{2}+2 a \xi\right)^{1 / 2}} F_{0}\left(\sqrt{E^{2}+2 a \xi}\right),
$$

a result identical to Equation (23). Finally, using the relation $N(E, \xi) \equiv F(E, \xi) / v(E)=\sqrt{m_{e} / 2}(F(E, \xi) / \sqrt{E})$, we readily recover Equation (15):

$$
\begin{aligned}
N(E, \xi) & =\sqrt{\frac{E}{E_{0}}}\left[\sqrt{\frac{m_{e}}{2}} \frac{F_{0}\left(E_{0}\right)}{\sqrt{E_{0}}}\right]=\sqrt{\frac{E}{E_{0}}} N_{0}\left(E_{0}\right) \\
& =\frac{E^{1 / 2}}{\left(E^{2}+2 a \xi\right)^{1 / 4}} N_{0}\left(\sqrt{E^{2}+2 a \xi}\right) .
\end{aligned}
$$

\subsection{Comparison with Numerical Results}

DZ claim that their "new solution" (10) of the continuity equation $(\mathrm{CE})$ is more consistent with numerical FP results than the existing analytical solution (15), stating that "the steeper positive slopes in the electron differential density obtained for the updated CE solutions very closely resemble the numeric solutions obtained from the FP approach" and citing Siversky \& Zharkova (2009). Since DZ's "solution" is mathematically incorrect, any improved consistency with numerical results must be purely coincidental. Nevertheless, to emphasize the validity of earlier results (Brown 1971; Syrovatskii \& Shmeleva 1972), we here address briefly the comparison with numerical results presented by DZ.

Driven by the term $\exp \left(-a \xi / 2 E^{2}\right)$ in Equation (10), all of the DZ solutions for the local electron distribution (their Figure 1) flatten and turn over from a power-law shape at high energies to a positive slope at low energies $\lesssim \sqrt{2 a \xi}$. DZ argue that this exponentially steep positive slope at low energies "can be easily accountable" for the generation of Langmuir waves found in numerical simulations. However, the correct solution for $N(E, \xi)$ (Equation (15)) also has a positive slope at low energies (driven by the $E^{1 / 2}$ term). It too produces a local maximum in $E$ around $E \sim \sqrt{2 a \xi}$ and explains the generation of Langmuir waves (Emslie \& Smith 1984). Hence the presence of Langmuir waves in numerical treatments in no way favors the DZ "solution" (10) over the existing solution (15).

DZ do not provide a direct comparison of their analytical solution with numerical results for the electron density distribution as a function of electron energy and column density. The only direct comparison with numerical results they provide is a comparison (their Figure 3) of spatially integrated X-ray spectra computed, for two different injected flux values and power-law spectral indices, with three electron distribution functions:

1. the existing (1D) solution (15);

2. their "updated" (1D) distribution function (10); and

3 . the electron distribution obtained from numerical FP calculations that include both collisional losses and pitch-angle scattering.

It must be noted that the numerical distribution (3) results from a $1.5 \mathrm{D}$ calculation and therefore is not directly comparable to the 1D analytical solutions (1) and (2). Although the impact of pitch-angle scattering on spatially integrated hard X-ray spectra is typically small (see, e.g., Leach \& Petrosian 1983; Massone et al. 2004), it can be significant enough to explain the differences in the solutions. Notwithstanding this caveat, the differences amongst the three results (Figure 3 of DZ) are less than a factor of $\sim(2-3)$ over the one order of magnitude in photon energy (10-100 keV) plotted. And (perhaps not surprisingly!) Figure 3 of DZ shows that the correct analytical solution (15) in many cases corresponds to the FP solutions better than the "new" DZ solution (10).

\section{CONCLUSIONS}

To sum up, the results of Dobranskis \& Zharkova (2014) are fundamentally incorrect because of an error made in solving the (coupled) ordinary differential Equations (5) through (7) obtained from application of the method of characteristics to the continuity equation for the electron number density (Equations (2) and (4)). Indeed, it is easily demonstrated that the DZ result (10) is not a solution of the continuity equation (4). When this mathematical error is noted and straightforwardly corrected, the well-established 1D analytical solution (15) for the density distribution function is recovered.

In conclusion, the validity of existing results, whether derived by the formalism of Syrovatskii \& Shmeleva (1972, et seq.), or the equivalent formalism of Brown (1971, et seq.), is confirmed. We strongly urge use of the long-established, correct solution in future works.

Useful discussions with Professor Ian Craig are gratefully acknowledged. A.G.E. was supported by NASA Grant NNX10AT78J. G.D.H. was supported by NASA Heliophysics Guest Investigator and Living with a Star TR\&T Grants and the RHESSI project. We thank the referee for suggesting the inclusion of the material in Section 2.2 and the editor for suggesting that two independently submitted papers with similar content be combined into the current work. 


\section{REFERENCES}

Brown, J. C. 1971, SoPh, 18, 489

Brown, J. C. 1972, SoPh, 26, 441

Craig, I. J. D., MacKinnon, A. L., \& Vilmer, N. 1985, Ap\&SS, 116, 377

Dobranskis, R. R., \& Zharkova, V. V. 2014, ApJ, 788, 42 (DZ)

Emslie, A. G. 1978, ApJ, 224, 241

Emslie, A. G., Barrett, R. K., \& Brown, J. C. 2001, ApJ, 557, 921

Emslie, A. G., \& Smith, D. F. 1984, ApJ, 279, 882
Holman, G. D., Aschwanden, M. J., Aurass, H., et al. 2011, SSRv, 159, 107

Holman, G. D., Sui, L., Schwartz, R. A., \& Emslie, A. G. 2003, ApJL, 595, L97

Kontar, E. P., Brown, J. C., Emslie, A. G., et al. 2011, SSRv, 159, 301

Leach, J., \& Petrosian, V. 1981, ApJ, 251, 781

Leach, J., \& Petrosian, V. 1983, ApJ, 269, 715

Massone, A. M., Emslie, A. G., Kontar, E. P., et al. 2004, ApJ, 613, 1233

McClements, K. G. 1992, A\&A, 258, 542

Siversky, T. V., \& Zharkova, V. V. 2009, A\&A, 504, 1057

Syrovatskii, S. I., \& Shmeleva, O. P. 1972, SvA, 16, 273 